Research Paper

\title{
Influence of Glucose Dosage in Parenteral Nutrition on Body Thiamine Levels in Rats
}

\author{
Daisuke Harada $^{1 凶}$, Mitsuo Nakayama² \\ 1. Laboratory of Clinical Nutrition, Naruto Research Institute, Otsuka Pharmaceutical Factory, Inc., 115 Kuguhara, Tateiwa, Muya-cho, Naruto, Tokushima \\ 772-8601, Japan \\ 2. PMM Group, Sales Division, Otsuka Pharmaceutical Factory, 2-9 Kanda Tsukasamachi, Chiyoda-ku, Tokyo 101-0048, Japan \\ $\square$ Corresponding author: Daisuke Harada, Laboratory of Clinical Nutrition, Naruto Research Institute, Otsuka Pharmaceutical Factory, Inc., 115 Kuguhara, \\ Tateiwa, Muya-cho, Naruto, Tokushima 772-8601, Japan. E-mail: Harada.Daisuke@otsuka.jp \\ (c) Ivyspring International Publisher. This is an open access article distributed under the terms of the Creative Commons Attribution (CC BY-NC) license \\ (https://creativecommons.org/licenses/by-nc/4.0/). See http://ivyspring.com/terms for full terms and conditions.
}

Received: 2018.07.25; Accepted: 2018.10.18; Published: 2019.01.01

\begin{abstract}
The objective of this study was to determine the relationship between glucose dosage in parenteral nutrition and reductions in levels of body thiamine in rats. Vitamin-free infusions with differing amounts of glucose were administered to normal or thiamine-deficient rats for 5 days, after which urinary thiamine excretion and the amounts of thiamine in the blood, liver, brain, and skeletal muscles were measured. The total energy dosage was set at three levels $(98,140$, and $196 \mathrm{kcal} / \mathrm{kg})$, and the dose of amino acids was constant among all groups. Urinary thiamine excretions on Day 5 decreased with increasing glucose dosage in the infusions. In normal rats, the amount of thiamine in the blood and all organs decreased compared with the diet group; however, no significant differences were found among the infusion groups. In thiamine-deficient rats, on the other hand, the amount of thiamine in the liver and skeletal muscles did not differ significantly among infusion groups; however, the amount of thiamine in the brain and blood decreased with increasing glucose dosage. An organ-specific correlation was found between glucose dosage in infusions and reductions in levels of thiamine. To prevent thiamine deficiencies from affecting the central nervous system, greater caution must be exercised during high-caloric parenteral nutrition. However, a constant supply of thiamine seemed to be essential, irrespective of the amount of energy supplied via parenteral nutrition, to maintain a sufficient level of thiamine in the body.
\end{abstract}

Key words: thiamine, vitamin $B_{1}$, parenteral nutrition, glucose, deficiency

\section{Introduction}

Thiamine (vitamin $B_{1}$ ) is a vitamin that is essential in energy-producing metabolic pathways, such as the glycolysis-tricarboxylic acid cycle, and represents the most important among various vitamins used in parenteral nutrition $(\mathrm{PN})$ that contains glucose as the major source of energy. Thiamine deficiencies can lead to serious outcomes, including Wernicke's encephalopathy and beriberi with lactic acidosis [1,2]. Therefore, administration of a sufficient amount of thiamine is necessary during PN. In recent years, cases of thiamine deficiency with PN have been reported [3-10].

In PN, the amounts of glucose, amino acids, and fats administered are determined according to the patient's nutritional state, disease, and duration of treatment. Used in patients who have lost intestinal function and other patients, total parenteral nutrition (TPN) includes large amounts of glucose as a source of energy, whereas peripheral parenteral nutrition (PPN), which is used in nutrition management for a short period of time, has relatively low glucose dosage because of the limitations on the osmotic pressure of the dosing liquid.

Since the publication of the WHO Report in 1965 [11], the practice has been to express thiamine requirements as amounts per human energy intake. Based on this concept, the requirement for thiamine in $\mathrm{PN}$ is interpreted as being higher with TPN, which involves greater amounts of glucose administered. In fact, in Japan, the attending physician is obliged to administer thiamine with TPN as mentioned in a Dear Doctor Letter (Urgent Safety Information No. 97-2) 
from the Ministry of Health and Welfare (currently the Ministry of Health, Labour and Welfare). However, with regard to PPN, no notifications like those for TPN have been issued by the administrative authorities, although the guideline from the Japanese Society for Parenteral and Enteral Nutrition emphasizes the necessity of administration of thiamine.

The aforementioned thiamine deficiencies during PN have been reported not only with TPN, but also with PPN [4,9]. The onset of thiamine deficiencies during $\mathrm{PN}$ is considered to depend largely on the presence of underlying nutrition disorders in post-gastrectomy patients [12], in patients following obesity surgery [13], in patients with eating disorders [14], and other patients, rather than on the amount of energy supplied in the infusion and the length of the treatment period. However, when discussed from another viewpoint, PPN is based on low-energy infusions; therefore, the fact that the administration of thiamine is likely to be neglected can also be contributory.

The 1965 WHO Report stated that "Although it has proved practical to tie the requirements of thiamine, riboflavin and niacin to caloric needs, more research is needed to learn whether, at very high and low levels of caloric consumption there is a good correlation as has been claimed for the medium, more ordinary ranges of energy output" [11]. Sauberlich et al. indicated that $0.3 \mathrm{mg}$ of thiamine per $1000 \mathrm{kcal}$ is necessary to maintain urinary thiamine excretion and erythrocyte transketolase activity by administering different caloric diet to young healthy subjects. However, the calorie dosage in that study was 2800 $\mathrm{kcal}$ or $3600 \mathrm{kcal}$ per day, either sufficient or excessive [15]. On the other hand, even when caloric intake is low, urinary excretion of thiamine continues and it is reported to cause thiamine deficiency [16, 17]. However, these cases were under fasting or extremely limited caloric intake. Therefore, it is difficult to extrapolate from these studies to predict the change in caloric intake and thiamine consumption in more usual caloric range.

In this study, parenteral nutritional infusions with differing amounts of glucose were administered to rats and post-dose urinary thiamine and levels of thiamine in the body were measured, and the relationship between glucose dosage and thiamine reductions was evaluated.

\section{Methods and Materials}

\section{Materials}

Commercially available vitamin-free infusions prepared for PPN or TPN containing glucose, amino acids and electrolytes were used in this study. In addition, a mixture of a PPN infusion with 50\% glucose solution $(\mathrm{PPN}+\mathrm{G})$ was prepared in order to administer energy at an intermediate level between PPN and TPN. The compositions of these infusions are shown in Table 1.

Table 1. Composition of test infusions

\begin{tabular}{llll}
\hline & PPN & PPN+G & TPN \\
\hline Volume $(\mathrm{mL})$ & 1000 & 1090 & 1100 \\
Glucose $(\%, \mathrm{w} / \mathrm{v})$ & 7.5 & 11.0 & 16.4 \\
Amino acids* $(\%, \mathrm{w} / \mathrm{v})$ & 3.00 & 2.75 & 2.73 \\
Total calories (kcal) & 420 & 600 & 840 \\
\hline PPN, peripheral parenteral nutrition; G, glucose; & TPN, total parenteral nutrition. \\
Commercially available vitamin-free infusions prepared for PPN or TPN \\
containing glucose, amino acids, and electrolytes were used. A mixture of a PN \\
infusion with 50\% glucose solution (PPN+G) was prepared in order to administer \\
energy at an intermediate level between PPN and TPN. \\
* Composed of 18 essential and non-essential amino acids.
\end{tabular}

\section{Animals}

Male Sprague-Dawley strain rats (8-wks-old for Experiment 1, and 6-wks-old for Experiment 2) were purchased from Charles River Japan, Inc. (Yokohama, Japan). Total 80 animals were acclimatized and each 10 animals were incorporated into each experimental group. In infusion group, total 6 animals were excluded from analysis because the test solution could not administer completely owing to catheter damage. All procedures were approved by the Committee for the Care and Use of Laboratory Animals of Otsuka Pharmaceutical Factory, Inc.

\section{Experiment 1 (normal rats)}

After being fed a standard diet (AIN-93M, Nosan Corporation, Yokohama, Japan) for 3 days, 10-week-old rats were divided into three groups: PPN, PPN+G and TPN. Infusions were administered for 5 days via a catheter placed in the external jugular vein. Daily energy dosages for PPN, PPN+G and TPN groups were 98, 140 and $196 \mathrm{kcal} / \mathrm{kg}$, respectively. These energy dosages are seven times higher than dosages for clinical use because the basal metabolic rate of rats is approximately seven times higher than that of humans, and each correspond to body weights of 840,1200 and $1680 \mathrm{kcal} / 60 \mathrm{~kg}$ in humans, respectively. Among the three infusion groups, the amino acid dosages were the same, and the only difference was the dosage of glucose (Table 2). Urine was collected on Days 1 and 5 of administration and a blood sample was collected from the caudal vena cava immediately after the end of infusion, after which the liver, brain and gastrocnemius (skeletal muscles) were then excised. The same samples were also collected from rats fed a standard diet for 3 days (Diet group). 
Table 2. Nutrient dosage for each experimental group

\begin{tabular}{|c|c|c|c|c|}
\hline & PPN & $\mathrm{PPN}+\mathrm{G}$ & TPN & Diet* \\
\hline Volume (mL) & 233 & 254 & 257 & - \\
\hline Glucose (g) & 17.5 & 27.9 & 42.1 & 41.1 \\
\hline Amino acids (g) & 7.0 & 7.0 & 7.0 & 8.0 \\
\hline Lipids (g) & 0.0 & 0.0 & 0.0 & 2.3 \\
\hline Total calories (kcal) & 98 & 140 & 196 & 217 \\
\hline Thiamine (g) & 0.00 & 0.00 & 0.00 & 0.27 or 0.00 \\
\hline
\end{tabular}

PPN, peripheral parenteral nutrition; G, glucose; TPN, total parenteral nutrition. The daily energy dosages for the PPN, PPN+G, and TPN groups were 98, 140, and $196 \mathrm{kcal} / \mathrm{kg}$, respectively. These energy dosages are seven times higher than the dosages for clinical use because the basal metabolic rate of rats is approximately seven times higher than that of humans, and correspond to body weights of 840 1200 , and $1680 \mathrm{kcal} / 60 \mathrm{~kg}$ in humans, respectively. Among the three infusion groups, the amino acid dosages were the same, and the only difference was the amount of glucose. Values are given as $\mathrm{kg} \mathrm{BW}^{-1} \bullet \mathrm{day}^{-1}$. ${ }^{*}$ Estimated from an assumption that a rat with a body weight of $350 \mathrm{~g}$ consumes $20 \mathrm{~g}$ of an AIN-93M diet or $20 \mathrm{~g}$ of a thiamine-deficient AIN-93M diet.

\section{Experiment 2 (thiamine-deficient rats)}

After being fed a thiamine-free diet (AIN-93M that was prepared with a specially ordered AIN-93 vitamin mixture not containing thiamine- $\mathrm{HCl}$, Nosan Corporation, Yokohama, Japan) for 14 days, 10-week-old rats were divided into three groups in the same manner as Experiment 1: PPN, PPN+G and TPN. Infusions and sample collections were carried out in the same manner as in Experiment 1. The same samples were also collected from rats fed a thiamine-free diet for 14 days (Deficient-diet group).

\section{Measurement}

A portion of each blood sample was promptly mixed with EDTA-2Na and then deproteinized using trichloroacetic acid.

Immediately after collection, outside left lobe of liver was perfused with ice-cold saline and then dehydrates and minced with scissors. Whole of brain and gastrocnemius were minced with scissors. 1.5-2 volumes (liver, brain) or 3-6 volumes (gastrocnemius) of cold pure water was added to the minced tissue and homogenized by using polytron homogenizer. Then trichloroacetic acid was added to homogenate, mixed and centrifuged and supernatant was retrieved. Each urine sample was stirred while adding trichloroacetic acid solution.

Each trichloroacetic acid-treated sample was centrifuged after treating the supernatant with Taka-Diastase to convert the phosphorylatedthiamine (thiamine monophosphate, thiamine diphosphate, thiamine triphosphate) into free thiamine, and total thiamine concentrations were measured by high-performance liquid chromatography with precolumn derivatization with thiochrome [18]. The amount of thiamine in the blood, liver, brain, and skeletal muscles were calculated using the following equations:
Amount of thiamine in the blood $(\mu \mathrm{g})=$ blood thiamine concentration $(\mu \mathrm{g} / \mathrm{mL}) \times$ body weight $(\mathrm{g}) \times$ $0.064(\mathrm{~mL} / \mathrm{g})$

Amount of thiamine in the liver $(\mu \mathrm{g})=$ liver thiamine concentration $(\mu \mathrm{g} / \mathrm{g}$ wet tissue $) \times$ liver weight $(\mathrm{g})$

Amount of thiamine in the brain $(\mu \mathrm{g})=$ brain thiamine concentration $(\mu \mathrm{g} / \mathrm{g}$ wet tissue $) \times$ brain weight $(\mathrm{g})$

Amount of thiamine in skeletal muscles $(\mu \mathrm{g})=$ gastrocnemius thiamine concentration $(\mu \mathrm{g} / \mathrm{g}$ wet tissue) $\times$ body weight $(\mathrm{g}) \times 0.04$

Yet another portion of each blood sample was treated with heparin and centrifuged to obtain plasma. The resulting plasma sample was subjected to various biochemical tests using an automatic analyzer 7170 (Hitachi High Technologies, Tokyo, Japan).

\section{Statistical analysis}

All data are presented as means and standard deviation. Tukey's multiple comparison test was used to compare the PPN, PPN+G and TPN groups, and Dunnett's multiple comparison test was used to compare the Pre group with each infusion group. The level of significance was set at $\mathrm{P}<.05$. Statistical analyses were performed using SAS version 8.02 (SAS Institute Japan Ltd., Tokyo, Japan), and EXSAS version 6.10 (Arm Systex, Osaka, Japan) was used for computations in Microsoft Excel.

\section{Results}

\section{Changes in body weight}

Table 3 shows rat body weights obtained before and after infusions and the corresponding percent changes. In both normal rats (Exp. 1) and thiamine-deficient rats (Exp. 2), post-infusion body weight decreased in the PPN group, decreased slightly in the PPN + G group, and increased in the TPN group, compared with baseline. Obvious differences in percent changes in body weight in each infusion group were not found between normal rats and thiamine-deficient rats.

\section{Urinary thiamine excretions}

Table 4 shows urinary thiamine excretions on Days 1 and 5 of the infusion. In normal rats, urinary thiamine excretions on Day 5 decreased to about one-tenth of levels on Day 1 in all groups. A comparison of the infusion groups showed that urinary thiamine excretions on Day 5 decreased with increasing glucose dosage in the infusion, with significantly lower excretions observed in the TPN group compared with the PPN group. 
Table 3. Body weights pre- and post-administration

\begin{tabular}{|c|c|c|c|}
\hline Normal rats & PPN $(n=10)$ & $\mathrm{PPN}+\mathrm{G}(\mathrm{n}=9)$ & TPN $(n=8)$ \\
\hline Pre $(g)$ & $363 \pm 23$ & $361 \pm 23$ & $360 \pm 31$ \\
\hline Post (g) & $342 \pm 25$ & $356 \pm 25$ & $366 \pm 33$ \\
\hline Change* (\%) & $\begin{array}{r}-5.8 \pm 2.0 \\
L_{\#}\end{array}$ & $\begin{aligned} &-1.7 \pm 1.4 \\
& \\
&\end{aligned}$ & $\begin{array}{l}1.8 \pm 1.9 \\
-ل 1\end{array}$ \\
\hline Thiamine-deficient rats & PPN (n=9) & $\mathrm{PPN}+\mathrm{G}(\mathrm{n}=10)$ & TPN $(\mathrm{n}=8)$ \\
\hline Pre (g) & $358 \pm 28$ & $359 \pm 21$ & $357 \pm 33$ \\
\hline Post (g) & $345 \pm 30$ & $356 \pm 25$ & $361 \pm 29$ \\
\hline Change* $(\%)$ & 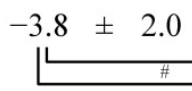 & $-0.8 \pm 1.9$ & $\underbrace{1.0}_{\#} \pm 3.4$ \\
\hline
\end{tabular}

PPN, peripheral parenteral nutrition; G, glucose; TPN, total parenteral nutrition.

Rat body weights obtained before and after infusions and the corresponding percent changes are shown as mean $\pm \mathrm{SD}$. ${ }^{*} \mathrm{Calculated}$ as $(\mathrm{BW}[\mathrm{post}]-\mathrm{BW}[\mathrm{pre}]) / \mathrm{BW}[\mathrm{pre}] \times 100$. \# $\mathrm{P}$ $<.05$ (Tukey's test)

Table 4. Excretion of thiamine in urine

\begin{tabular}{|c|c|c|c|}
\hline \multirow[b]{3}{*}{ Normal rats } & \multicolumn{3}{|c|}{ Urinary thiamine ( $\mu \mathrm{g} /$ day $)$} \\
\hline & & Day 1 & Day 5 \\
\hline & $\operatorname{PPN}(\mathrm{n}=10)$ & $18.1 \pm 3.9$ & $2.11 \pm 0.197$ \\
\hline & $\mathrm{PPN}+\mathrm{G}(\mathrm{n}=9)$ & $18.6 \pm 3.8$ & $1.77 \pm 0.30$ \\
\hline & TPN $(n=8)$ & $18.3 \pm 4.6$ & $1.52 \pm 0.45\rfloor$ \\
\hline \multirow[t]{3}{*}{ Thiamine-deficient rats } & PPN (n=9) & $0.73 \pm 0.14$ & $0.35 \pm 0.22$ \\
\hline & $\mathrm{PPN}+\mathrm{G}(\mathrm{n}=10)$ & $0.75 \pm 0.20$ & $0.27 \pm 0.19$ \\
\hline & TPN $(n=8)$ & $0.80 \pm 0.24$ & $\mathrm{NC}$ \\
\hline
\end{tabular}

PPN, peripheral parenteral nutrition; G, glucose; TPN, total parenteral nutrition.

Urinary thiamine excretions on Days 1 and 5 of the infusion are shown as mean \pm SD. NC: Not calculated because thiamine concentrations were below the detection limit in 4 of the 8 samples. \# $\mathrm{P}<.05$ (Tukey's test).

In thiamine-deficient rats, urinary thiamine excretions were already low on Day 1, and decreased further on Day 5. On Day 5, urinary thiamine concentrations in 4 out of the 8 animals in the TPN group were below the limit of quantitation and the excretions tended to decrease with increasing glucose dosage in the infusion as in the normal rats.

\section{Amount of thiamine in the blood and organs}

Table 5 shows the amount of thiamine in the blood, liver, brain, and skeletal muscles on Day 5 .

In normal rats, the amount of thiamine in the blood and all organs decreased significantly or tended to decrease in the infusion groups compared with the diet group which represents thiamine status of at the start of infusion; however, no significant differences were observed among the infusion groups.

Likewise, in thiamine-deficient rats, the amount of thiamine in the blood and all organs (which were already at low levels at the start of administration) decreased further after 5-day infusions. A comparison of the infusion groups showed that no significant differences in the amount of thiamine in the liver and skeletal muscles were observed. In the blood and brain, however, the amount of thiamine decreased with increasing glucose dosage in the infusion, with significantly lower values obtained in the TPN group than in the PPN group.

\section{Blood chemistry}

Table 6 shows blood chemistry values. Although blood glucose levels did not differ among the infusion groups in both normal rats and thiamine-deficient rats, lactic acid and pyruvic acid increased with increasing glucose dosage in the infusion in thiamine-deficient rats, with significantly higher values for both parameters obtained in the TPN group than in the PPN group. 
Table 5. Amounts of thiamine in the blood and organs

\begin{tabular}{|c|c|c|c|c|c|c|c|c|c|c|c|}
\hline \multirow{3}{*}{ Normal rats } & \multirow[b]{3}{*}{$\operatorname{Diet}(\mathrm{n}=10)$} & \multicolumn{10}{|c|}{ Thiamine $(\mu \mathrm{g})$} \\
\hline & & \multicolumn{3}{|c|}{ Blood } & \multicolumn{3}{|c|}{ Liver } & \multicolumn{3}{|c|}{ Brain } & $\begin{array}{l}\text { Skeletal } \\
\text { muscles }\end{array}$ \\
\hline & & 6.03 & \pm & 0.82 & 53.8 & \pm & 8.5 & 2.76 & \pm & 0.20 & $188 \pm 35$ \\
\hline & PPN $(n=10)$ & 2.59 & \pm & $0.55^{*}$ & 15.9 & 1 & $2.6^{*}$ & 2.36 & \pm & $0.22^{*}$ & $134 \pm 19^{*}$ \\
\hline & $\mathrm{PPN}+\mathrm{G}(\mathrm{n}=9)$ & 2.41 & \pm & $0.48^{*}$ & 15.1 & $\perp$ & $2.8^{*}$ & 2.55 & \pm & 0.27 & $141 \pm 21^{*}$ \\
\hline & TPN $(n=8)$ & 2.31 & \pm & $0.23^{*}$ & 13.9 & \pm & $2.3^{*}$ & 2.42 & \pm & $0.23^{*}$ & $159 \pm 28$ \\
\hline \multirow[t]{4}{*}{ Thiamine-deficient rats } & Deficient-diet $(\mathrm{n}=10)$ & 0.69 & \pm & 0.08 & 6.2 & \pm & 0.8 & 1.88 & \pm & 0.22 & $50 \pm 8$ \\
\hline & $\operatorname{PPN}(n=9)$ & 0.47 & \pm & $0.07^{*} \square$ & 2.3 & \pm & $0.7^{*}$ & 1.57 & \pm & $0.15^{*} 7$ & $35 \pm 7^{*}$ \\
\hline & $\mathrm{PPN}+\mathrm{G}(\mathrm{n}=10)$ & 0.41 & \pm & $\left.0.07^{*}\right\rceil_{\#}$ & 2.2 & \pm & $0.5^{*}$ & 1.40 & \pm & $0.18^{*}$ & $33 \pm 6^{*}$ \\
\hline & TPN $(n=8)$ & 0.32 & \pm & $\left.0.05^{*}\right]^{\prime \prime}$ & 2.2 & \pm & $0.4^{*}$ & 1.31 & \pm & $\left.0.14^{*}\right]$ & $35 \pm 5^{*}$ \\
\hline
\end{tabular}

PPN, peripheral parenteral nutrition; G, glucose; TPN, total parenteral nutrition.

Values in the diet group represent thiamine amounts at the start of infusion in each experiment. Amounts of thiamine on Day 5 are shown as mean \pm SD. Amounts of thiamine in the blood, liver, brain, and skeletal muscles were calculated using the following equations: Amount of thiamine in the blood $(\mu \mathrm{g})=\mathrm{blood}$ thiamine concentration $(\mu \mathrm{g} / \mathrm{mL}) \times$ body weight $(\mathrm{g}) \times 0.064(\mathrm{~mL} / \mathrm{g})$; Amount of thiamine in the liver $(\mu \mathrm{g})=$ liver thiamine concentration $(\mu \mathrm{g} / \mathrm{g}$ wet tissue $) \times$ liver weight $(\mathrm{g})$; Amount of thiamine in the brain $(\mu \mathrm{g})=$ brain thiamine concentration $(\mu \mathrm{g} / \mathrm{g}$ wet tissue $) \times$ brain weight $(\mathrm{g})$; Amount of thiamine in skeletal muscles $(\mu \mathrm{g})=\mathrm{gastrocnemius}$ thiamine concentration $(\mu \mathrm{g} / \mathrm{g}$ wet tissue $) \times$ body weight $(\mathrm{g}) \times 0.04$

${ }^{*} \mathrm{P}<.05$ vs. diet group in each experiment (Dunnett's test). ${ }^{*} \mathrm{P}<.05$ among the three infusion groups (Tukey's test).

Table 6. Blood chemistry data

\begin{tabular}{|c|c|c|c|c|c|c|c|c|c|c|}
\hline \multirow[b]{2}{*}{ Normal rats } & \multirow[b]{2}{*}{$\operatorname{Diet}(n=10)$} & \multicolumn{2}{|c|}{ Glucose (mg/dL) } & \multicolumn{4}{|c|}{$\mathrm{LA}(\mathrm{mg} / \mathrm{dL})$} & \multicolumn{3}{|c|}{$\mathrm{PA}(\mathrm{mg} / \mathrm{dL})$} \\
\hline & & 180 & \pm 17 & 21.0 & \pm & 4.7 & & 1.5 & \pm & 0.3 \\
\hline & $\mathrm{PPN}(\mathrm{n}=10)$ & 132 & $\pm 14^{*}$ & 27.7 & \pm & 6.3 & & 1.8 & \pm & 0.3 \\
\hline & $\mathrm{PPN}+\mathrm{G}(\mathrm{n}=9)$ & 128 & $\pm 8^{*}$ & 27.7 & \pm & 6.5 & & 1.8 & \pm & 0.3 \\
\hline & TPN (n=8) & 124 & $\pm 13^{*}$ & 36.0 & \pm & $10.8^{*}$ & & 2.1 & \pm & $0.4^{*}$ \\
\hline \multirow[t]{4}{*}{ Thiamine-deficient rats } & Deficient-diet $(n=10)$ & 175 & \pm 11 & 22.3 & \pm & 5.1 & & 1.8 & \pm & 0.4 \\
\hline & $\operatorname{PPN}(n=9)$ & 142 & $\pm 13^{*}$ & 29.8 & \pm & 4.8 & & 2.2 & \pm & 0.4 \\
\hline & $\mathrm{PPN}+\mathrm{G}(\mathrm{n}=10)$ & 147 & $\pm 10^{*}$ & 34.1 & \pm & $5.0^{*}$ & \# & 2.6 & \pm & $0.4^{*}$ \\
\hline & TPN $(n=8)$ & 140 & $\pm 29^{*}$ & 42.0 & \pm & $12.6^{*}$ & & 3.4 & \pm & $\left.1.5^{*}\right\rfloor$ \\
\hline
\end{tabular}

LA, lactic acid; PA, pyruvic acid; PPN, peripheral parenteral nutrition; G, glucose; TPN, total parenteral nutrition.

Blood chemistry values on Day 5 are shown as mean $\pm \mathrm{SD}$. ${ }^{*} \mathrm{P}<.05$ vs. diet group in each experiment (Dunnett's test). \# $\mathrm{P}<.05$ among the three infusion groups (Tukey's test).

\section{Discussion}

With regard to human thiamine requirements, the 2015 Edition of the Dietary Reference Intakes for Japanese [19] specifies the estimated average requirement for thiamine as $0.35 \mathrm{mg} / 1000 \mathrm{kcal}$ in reference to the WHO Report. The Reference Nutrient Intake in the UK is $0.4 \mathrm{mg} / 1000 \mathrm{kcal}$ [20]. However, the 1965 WHO Report also stated that "Although it has proved practical to tie the requirements of thiamine, riboflavin and niacin to caloric needs, more research is needed to learn whether, at very high and low levels of caloric consumption there is a good correlation as has been claimed for the medium, more ordinary ranges of energy output" [11]. In the past, the relationship between caloric intake and thiamine consumption has been investigated in some studies, but they were conducted under the caloric range of moderately to excess [15], or extremely low range [16, 17]. As far as we know, no studies have conducted to reveal the relationship between caloric intake and thiamine consumption in more usual caloric range.

In this study, parenteral nutritional infusion was administered to rats at differing amounts of glucose, after which the amount of thiamine in the body was measured. Urinary thiamine excretions, a sensitive marker of body thiamine depletion [21], decreased with increasing glucose dosage in the infusion and, among the thiamine-deficient rats, rats in the TPN group that received a higher dosage of glucose had lower amounts of thiamine in the blood and brain. In normal rats, however, the amount of thiamine in the blood and all organs was not influenced by the dosage of glucose in the infusion. In thiamine-deficient rats as well, the amount of thiamine in the liver and skeletal muscles, the major storages of body thiamine, were not influenced by the dosage of glucose.

McCourt et al. [22] showed that during the catalytic reaction of acetohydroxy acid synthase with thiamine diphosphate (ThDP) as a cofactor, the ThDP 
became instable, decomposed, and disappeared, and that pyruvate decarboxylase caused a similar phenomenon. McCourt et al. stated that although these enzymes are not found in mammals, a similar phenomenon occurs during the catalytic reaction of pyruvate dehydrogenase and oxoglutarate dehydrogenase involved in human glycometabolism, which represents a biochemical background for the increased thiamine requirements with carbohydrate ingestion. This hypothesis may well explain the reason why thiamine decreases in the brains of thiamine-deficient rats with increasing glucose dosage in infusions. Specifically, intracellular thiamine is known to be mostly present as ThDP bound to apoenzymes in the brain unlike other tissues [23], and little free thiamine may be present in cells in the thiamine-deficient state. Therefore, the relationship between the metabolic load on apoenzymes with increased glucose dosage and the resulting thiamine decomposition may manifest clearly in the brain. The increased metabolic load on pyruvate dehydrogenase with increased glucose dosage manifested as an increase in blood lactic acid and pyruvic acid concentrations in the TPN group.

On the other hand, free thiamine in the cells are for the most part metabolized into a large number of decomposition products based on the action of detoxicating enzymes in the liver and kidneys, and then excreted in urine [23]. In the liver and skeletal muscles and, even in the brains of normal rats, free thiamine not bound to glycometabolizing enzymes was likely present in the cells. Therefore, the observed tissue thiamine reductions in these organs mainly reflected free thiamine decomposition by detoxicating enzymes, hence the influence of glucose dosage may be masked and may be difficult to observe.

Thiamine in erythrocytes has been reported to exist mostly as ThDP [24]. Therefore, also in erythrocytes, as with the brain, ThDP bound to apoenzyme such as transketolase may be degraded as glucose load increases, resulting in a decrease in blood thiamine amount. The influence of glucose dosage on urinary thiamine excretions is considered to be a phenomenon mediated by the influence on amounts of thiamine in the blood.

There are some limitations on this study. First, thiamin concentrations in blood, urine and tissues were measured as total thiamine concentration which is sum of the free thiamine, thiamine monophosphate, thiamine diphosphate (ThDP) and thiamine triphosphate. This is same method with clinical nutritional assessment examination which is supported with health insurance in Japan. However, as described above in the discussion, it is considered that the mechanism of the difference in thiamine decrease among blood and tissues could be analyzed in more detail by measuring the concentration of each phosphorylated thiamine, especially ThDP. Second, in this study, fat-free infusions were used for PPN and TPN because they are basic parenteral nutrition formulation in Japan. But it has been known that the level of intake fat may reduce the requirement for thiamine $[25,26]$. Fatty acid synthesis could be upregulated in the body during fat-free parenteral nutrition. In fatty acid synthesis, NADPH is required and which is supplied from the pentose phosphate pathway. In the pentose phosphate pathway, transketolase, which require ThDP as a cofactor, is one of the key enzymes which catalyze trans-carbon reaction between 5-carbon ketose and 5-carbon aldose. Thus, by the metabolic load onto transketolase activity which was accelerated accompanying to the fatty acid synthesis under the fat-free parenteral nutrition in this study, extra decrease of thiamine could be observed through ThDP degradation. If fat-containing infusions were used, the difference between groups of thiamine decrease may be smaller, as the contribution of pentose phosphate pathway in energy metabolism could be reduced.

The results of this study lead to two recommendations concerning thiamine replenishment in PN. One recommendation is that, when performing high-energy TPN for patients with suspected thiamine deficiency, thiamine replenishment and blood concentration monitoring must be planned more carefully by taking the fact that brain thiamine may be lost quickly under high-glucose loaded conditions into account. Recently, Suzuki et al. demonstrated that high-dose thiamine treatment prevents brain lesions and prolongs survival of SLC19A3-deficient mice [27]. SLC19A3 is the gene encoding thiamine transporter 2 and it's mutation is responsible for the thiamine metabolism dysfunction syndrome-2 (THMD2) which is an autosomal recessive neurodegenerative disorder. High-dose of thiamine may overcome intestinal absorption disorder derived from transporter dysfunction, followed by elevation of thiamine concentration in blood and brain. Therefore, in order to prevent brain damage due to thiamine deficiency during TPN, administration of higher doses of thiamine may be effective. The other recommendation is that thiamine content in the body as a whole is for the most part present in the liver and skeletal muscles, for which a certain amount is lost even in a short period of time irrespective of the dosage of glucose supplied in PN; therefore, it is necessary to constantly replenish the amount of thiamine to maintain the required sufficient amount. This supports for the old recommendation of the NRC-NAS Food and 
Nutrition Board that for adults with energy intakes of less than $2000 \mathrm{kcal}$ per day, an intake of $1 \mathrm{mg}$ per day of thiamine should be maintained [28].

In conclusion, this study demonstrated an organ-specific correlation between glucose dosage in PN and decreases in amounts of thiamine in the body. To prevent thiamine deficiencies from affecting the central nervous system, greater caution must be exercised during high-energy TPN. However, a constant supply of thiamine seemed to be essential, irrespective of the amount of energy supplied in PN, to maintain a sufficient amount of thiamine in the body.

\section{Abbreviations}

TPN: total parenteral nutrition; PPN: peripheral parenteral nutrition; ThDP: thiamine diphosphate.

\section{Acknowledgements}

The authors present special thanks for Dr. Akira Momii for his broad and general advice concerning to clinical nutrition.

\section{Competing Interests}

The authors have declared that no competing interest exists.

\section{References}

[1] Arts NJ, Walvoort SJ, Kessels RP. Korsakoff's syndrome: a critical review. Neuropsychiatr Dis Treat. 2017; 13: 2875-90.

[2] Numata G, Kodera S, Kiriyama H, et al. Usefulness of central venous saturation as a predictor of thiamine deficiency in critically ill patients: a case report. J Intensive Care. 2017; 5: 61. doi 10.1186/s40560-017-0255-7.

[3] Serin SO, Karaoren G, Okuturlar Y, et al. Thiamin and folic acid deficiency accompanied by resistant electrolyte imbalance in the re-feeding syndrome in an elderly patient. Asia Pac J Clin Nutr. 2017; 26(2): 379-82.

[4] Akiyama H, Saito M, Ohtsuka Y. A case of Wernicke's encephalopathy presenting with acute deterioration of consciousness caused by peripheral parenteral nutrition. No Shinkei Geka. 2015; 43(12): 1113-8. [Article in Japanese]

[5] Shin IS, Seok H, Eun YH, et al. Wernicke's encephalopathy after total parenteral nutrition in patients with Crohn's disease. Intest Res. 2016; 14(2): 191-6.

[6] Salvatori G, Mondì V, Piersigilli F, et al. Thiamine deficiency in a developed country: acute lactic acidosis in two neonates due to unsupplemented parenteral nutrition. JPEN. 2016; 40(6): 886-9.

[7] Barnes D, Kerner J. Severe lactic acidosis in a parenteral nutrition-dependent teenager with ulcerative colitis. Dig Dis Sci. 2016; 61(10): 2804-6.

[8] Da Silva YS, Horvat CM, Dezfulian C. Thiamin deficiency as a cause of persistent hyperlactatemia in a parenteral nutrition-dependent patient. JPEN. 2015; 39(5): 604-6.

[9] Ozasa H, Ishibashi N, Imaizumi $\mathrm{T}$, et al. Lactic acidosis due to peripheral parenteral nutrition after total gastrectomy-a case report. Nihon Rinsho Geka Gakkai Zasshi. 2008; 69: 761-6. [Article in Japanese]

[10] Francini-Pesenti F, Brocadello F, Manara R, et al. Wernicke's syndrome during parenteral feeding: not an unusual complication. Nutrition. 2009; 25(2): 142-6.

[11] [No authors listed]. Requirements of vitamin A, thiamine, riboflavin and niacin: report of a joint $\mathrm{FAO} / \mathrm{WHO}$ expert group. Geneva: World Health Organization; 1967.

[12] Iwase K, Higaki J, Yoon HE, et al. Reduced thiamine (vitamin B1) levels following gastrectomy for gastric cancer. Gastric Cancer. 2002; 5(2): 77-82.

[13] Ba F, Siddiqi ZA. Neurologic complications of bariatric surgery. Rev Neurol Dis. 2010; 7(4): 119-24.

[14] Winston AP, Jamieson CP, Madira W, et al. Prevalence of thiamin deficiency in anorexia nervosa. Int J Eat Disord. 2000; 28(4): 451-4.

[15] Sauberlich HE, Herman YF, Stevens CO, et al. Thiamin requirement of the adult human. Am J Clin Nutr. 1979; 32(11): 2237-48.
[16] Drenick EJ, Joven CB, Swendseid ME. Occurrence of acute Wernicke's encephalopathy during prolonged starvation for the treatment of obesity. $\mathrm{N}$ Engl J Med. 1966; 274(17): 937-9.

[17] Consolazio CF, Johnson HL, Krzywicki HJ, et al. Thiamin, riboflavin, and pyridoxine excretion during acute starvation and calorie restriction. Am J Clin Nutr. 1971; 24(9): 1060-7.

[18] Mino M. Standard method on vitamin determination for clinical samples, as recommended by the Vitamin Society of Japan. Vitamins. 2000; 74: 501-15. [Article in Japanese]

[19] Hishida A, Sasaki S. Dietary reference intakes for Japanese. Tokyo: Daiichi-Shuppan Publishing; 2015.

[20] [No authors listed]. Dietary reference values for food energy and nutrients for the United Kingdom: report of the panel on dietary reference values of the committee on medical aspects of food policy. Report on health and social subjects no. 41. London: HMSO; 1991.

[21] Fukuwatari T, Yoshida E, Takahashi K, et al. Effect of fasting on the urinary excretion of water-soluble vitamins in humans and rats. J Nutr Sci Vitaminol. 2010; 56(1): 19-26.

[22] McCourt JA, Nixon PF, Duggleby RG. Thiamin nutrition and catalysis-induced instability of thiamin diphosphate. Br J Nutr. 2006; 96(4): 636-8.

[23] Bettendorff L. Thiamine. In: Erdman JW Jr, Macdonald IA, Zeisel SH, ed. Present knowledge in nutrition 10th edition, Washington: ILSI Press; 2012: 261-79.

[24] Macinelli R, Ceccanti M, Guiducci MS, et al. Simultaneous liquid chromatographic assessment of thiamine, thiamine monophosphate and thiamine diphosphate in human erythrocytes: a study on alcoholics. J Chromatgr B. 2003; 789(2): 355-63.

[25] Ellis NR, Madsen LL. The thiamine requirement of pigs as related to the fat content of the diet. J Nutr. 1944; 27: 253.

[26] Balaghi $M$, Pearson WN. Alterations in the metabolism of 2-14C-thiazole-labeled thiamine by the rat induced by a high fat diet or thyroxine. J Nutr. 1966; 90(2): 161-6.

[27] Suzuki K, Yamada K, Fukuhara Y, et al. High-dose thiamine prevents brain lesions and prolongs survival of Slc19a3-deficient mice. PLoS ONE. 2017; 12(6): e0180279.

[28] [No authors listed]. Recommended Dietary Allowance ( $8^{\text {th }}$ ed.). Washington, D.C.: National Academy of Science-National Research Council; 1974 